A N N A L E S Annales de Bretagne et des Pays de l'Ouest

Anjou. Maine. Poitou-Charente. Touraine

123-4 | 2016

Varia

\title{
11 batailles qui ont fait la Bretagne
}

\section{Valeria Pansini}

\section{OpenEdition}

Journals

Édition électronique

URL : http://journals.openedition.org/abpo/3468

DOI : $10.4000 /$ abpo.3468

ISBN : 978-2-7535-5365-1

ISSN : 2108-6443

Éditeur

Presses universitaires de Rennes

Édition imprimée

Date de publication : 30 décembre 2016

Pagination : 205-207

ISBN : 978-2-7535-5363-7

ISSN : 0399-0826

Référence électronique

Valeria Pansini, «11 batailles qui ont fait la Bretagne », Annales de Bretagne et des Pays de l'Ouest [En ligne], 123-4 | 2016, mis en ligne le 30 décembre 2016, consulté le 23 septembre 2020. URL : http:// journals.openedition.org/abpo/3468; DOI : https://doi.org/10.4000/abpo.3468 
sacerdoce. Les vacances s'allongent (aux Gras et à la Toussaint à partir de 1955) mais il importe qu'en famille ou en " colo ", la surveillance du recteur ou le maintien de cadres collectifs de piété conserve le petit séminariste dans l'esprit de "sa " vocation.

L'optimisme est de rigueur au seuil des années 1960, à l'heure où s'élèvent enfin les murs de la chapelle définitive. Quand les élèves en creusent les fondations, au printemps 1957, la Voix du Petit Séminaire envisage l'avenir sans inquiétude : " J'étais là quand on a commencé, diront bon nombre de recteurs dans cinquante ans! " On le devine : très peu de ces élèves sont devenus prêtres et tous ne le sont pas " restés ". Mais en 1957, plus des trois quarts des élèves de terminale du Petit Séminaire vont encore au Grand et chaque promotion d'ordinands compte $60 \%$ d'anciens de Quintin... Comment imaginer alors que la promotion de $6^{\mathrm{e}}$ admise à la rentrée précédente sera la première à ne fournir aucun prêtre? D'autant que la nouvelle chapelle regarde résolument vers l'avenir : ses volumes, dessinés par le dominicain Cocagnac de la revue Art sacré, convergent vers l'autel, anticipant les réformes conciliaires. L'aspiration au changement semble alors irrépressible, tant elle semble correspondre aux désirs de la jeunesse : on pourra méditer, p. 171, sur cette photo de grands élèves posant derrière des barbelés en 1956... À partir de 1962 s'ouvre " le temps difficile des mutations ", que signale la plongée rapide des effectifs. Distincte de la chute des vocations, elle s'explique d'abord par la multiplication des CES pourquoi envoyer, dès lors, les garçons à Quintin? - et la désaffection des familles pour les pensionnats. Mais les responsables de la maison y voient un motif supplémentaire d'ouvrir largement l'établissement sur un monde qui bouge et que l'on veut envisager positivement, loin du " cocon " et de la "serre chaude " désormais récusés. Comme d'autres Petits Séminaires, Quintin s'avère donc très réceptif aux expérimentations nouvelles - du journal des élèves en 1962 à l'autodiscipline après 1968 - en raison inverse de la rigidité du modèle initial.

À partir de 1966, sous la direction du P. Colleter, la dimension vocationnelle devient de facto périphérique : "séminaire de jeunes " en 1966 puis " centre JeanXXIII " deux ans plus tard, Quintin devient surtout un collège-lycée catholique rayonnant avec succès sur le secteur alentour. Les contrats d'association avec l'État (1968 et 1970), l'accueil d'externes et la mixité (1971), la fusion avec les deux autres écoles catholiques de Quintin (1975), la laïcisation du corps enseignant (un directeur laïc en 1979) jalonnent les étapes d'une reconversion d'autant plus réussie qu'elle est rare parmi les anciens Petits Séminaires bretons (le lycée-collège de Sainte-Anne d'Auray offrirait cependant un autre exemple). Sans doute le lecteur aurait-il aimé que l'histoire retracée par Yves Moigno ouvre davantage de points de comparaison mais la bibliographie sur le sujet et le récent colloque consacré au Petit Séminaire de Sainte-Anne d'Auray (octobre 2015) permettront ici d'utiles extensions. Riche de témoignages sans apprêt, fort de données statistiques patiemment réunies et analysées, nourri des questions de la " génération de la rupture " avec ce mélange de distance et d'empathie qu'autorise le recul du temps, l'ouvrage d'Yves Moigno va, à l'évidence, bien au-delà des seuls anciens élèves.

Georges Provost

Le Page, Dominique (dir.), 11 batailles qui ont fait la Bretagne, Morlaix, Skol Vreizh, 2015,359 p.

L'ouvrage collectif dirigé par Dominique Le Page couvre, à travers les faits de guerre qui ont touché la région, deux millénaires d'histoire de la Bretagne, de 
l'affrontement entre la flotte romaine et les Vénètes du Morbihan en 56 av. J.-C. à la bataille du maquis de Saint Marcel en juin 1944. L'attention portée par les onze contributions à la mémoire de l'événement analysé rend ce très impressionnant laps de temps étudié encore plus long, en emmenant l'attention du lecteur jusqu'à nos jours, des célébrations des batailles passées aux exploitations muséographiques et touristiques de ces faits de guerre.

L'ouvrage a le mérite certain de proposer au lecteur, chercheur ou passionné d'histoire de Bretagne, une analyse historique établie avec rigueur. Dans chaque contribution, les récits des faits de guerre sont mis à l'épreuve. Le lecteur retrouve ainsi une critique précise des sources qui permettent (ou pas) de connaître le déroulement factuel des événements et une analyse lucide des enjeux de la construction de la mémoire de la bataille. Le schéma commun aux contributions, expliqué par Dominique Le Page dans son introduction (p. 15), prévoit que la présentation des sources utilisées soit suivie d'une reconstitution factuelle du déroulement de la bataille et que, dans un troisième temps, on s'attache à étudier la mémoire des événements " en s'intéressant à ceux qui ont contribué à la construire et à l'entretenir ". Lire avec la même grille des faits se déroulant sur deux millénaires d'histoire de la Bretagne est un défi : si le triple questionnement est effectivement utilisé dans pratiquement toutes les contributions, le plan n'est pas toujours régulièrement suivi et les chapitres sont trop différents en taille, des 11 pages sur la bataille de Ballon écrites par Yves Coativy (p. 34-45) aux 60 pages d'Alain Gallicé et Dominique Le Page sur Saint-Aubin-du-Cormier (p. 74-133) pour que l'effet encyclopédique, qui demanderait plus de régularité, puisse opérer.

L'unité de l'ensemble des contributions est construite, ou plutôt donnée pour acquise, sur base géographique : les batailles étudiées se sont déroulées en Bretagne ou peuvent être considérées comme " bretonnes " en raison de leur insertion dans " une situation politique et militaire régionale " (Hervé Le Goff à propos de la bataille de Craon en 1592, p. 155) ou à cause de la participation importante de combattants d'origine bretonne à l'événement et à la construction successive d'un " récit légendaire ", d'une " construction mémorielle érigée sur une historiographie très partielle " (p. 267) dans lesquels la composante bretonne est fondamentale : c'est le cas de la bataille de Dixmude (Belgique), efficacement étudiée par Jean-Christophe Fichou, qui s'interroge dès le titre - « La bataille de Dixmude (octobre-novembredécembre 1914) : une bataille bretonne? "-sur l'appartenance de ce fait de guerre à ceux " qui ont fait la Bretagne ". Paradoxalement, nous devons chercher les lignes de construction de l'unité thématique dans l'étude de ces batailles " excentrées ": la nécessité d'en motiver l'insertion dans le récit bimillénaire des onze batailles qui ont fait la Bretagne porte à expliciter les critères de cette construction d'unité, qui resteraient autrement cachés. Dans l'ensemble de l'ouvrage, l'unité géographique est donnée comme évidente, mais même si on ne questionne pas la permanence des limites de cette unité sur 2000 ans, il aurait été souhaitable de discuter davantage la portée historiographique de cette localisation. La continuité artificielle du " récit régional " (p. 18) et les "approximations " (p. 19) dont ce récit s'est nourri sont clairement expliquées dans l'introduction et régulièrement mises à l'épreuve de la rigueur historiographique dans les différentes contributions. Néanmoins, l'ouvrage rassemblant en ordre chronologique strict des études de faits de guerre, et le titre, 11 batailles qui ont fait la Bretagne, évoquant clairement l'apport de ces faits de guerre à la construction d'une identité bretonne, une réflexivité plus constante aurait été souhaitable.

La première bataille bretonne connue et traitée dans cet ouvrage est en réalité armoricaine : il s'agit de la bataille des Romains contre les Vénètes en 56 av. J.-C., étudiée par Jean-Yves Eveillard. La richesse du questionnement, l'attention aux 
conséquences de la bataille sur la population, comme elles peuvent être perçues à travers les sources écrites et les observations archéologiques, font de cette contribution un exemple de comment on peut à travers l'étude de la guerre percevoir la réalité historique des populations qui s'y engagent et questionner leurs structures sociales et leurs évolutions. Le parcours dans l'histoire de la Bretagne continue par Ballon, bataille étudiée par Yves Coativy, extrêmement critique envers le "roman breton ». L'auteur explique, de façon très intéressante, comment deux événements distincts mais proches géographiquement, la bataille de Ballon (845) et celle de Jengland-Beslé (851), ont fini par se contaminer (p. 36). La superposition des récits, avec celui des faits de Jengland-Beslé, plus récents et mieux documentés, en position dominante, "la répétition des informations erronées [qui] les transforme en vérité " (p. 45), ont créé une vision des événements largement orientée, où Ballon devient un point culminant de l'histoire bretonne : " la bataille fondatrice de la Bretagne médiévale, moderne et contemporaine " (p. 41). Les deux contributions suivantes, celle de Laurence Moal sur Auray (1364) et de Alain Gallicé et Dominique Le Page sur Saint-Aubin-du-Cormier (1488), sont très riches et complètes sur ces deux très célèbres batailles : les aspects techniques, tactiques, mais aussi mémoriels sont pris en compte et traités de façon exhaustive. On regrettera pourtant qu'il n'y ait pas davantage de dialogue entre le texte de Laurence Moal, qui évoque déjà les faits de Quiberon en 1795 et la contribution de Youenn Le Prat, qui est totalement consacrée à cette bataille. Plus généralement, les références en commun, parfois les sources communes à plusieurs contributions portant sur l'époque médiévale et moderne, auraient pu générer plus d'échanges entre les chapitres.

Pour l'époque moderne, la suite des batailles bretonnes est complétée par les débarquements dans la zone de Brest : la descente du Conquet en 1558, étudiée par Pol Vendeville ainsi que le combat de Camaret en 1694, analysé par Yann Lagadec et Stéphane Perréon. La documentation de Pol Vendeville est très riche et permet de questionner les motivations de cette descente en force sur les côtes bretonnes : prendre Brest, ou ravager le littoral pour pousser le roi de France à détourner ses troupes des autres fronts (p. 145)? Pour Camaret aussi, la question du véritable objectif de l'expédition reste importante, tant la prise de Brest apparaît ardue pour les assaillants. Yann Lagadec et Stéphane Pérréon mettent en évidence le fait que la stratégie du blocus, réalisée seulement dans les années 1740 , a ses origines dans la bataille de Camaret et l'abandon qui s'en suit du projet de prise du port (p. 213). Pour Camaret, au niveau mémoriel, « le monument - la tour Vauban - est venu comme effacer l'événement " (p. 221) : " la tour est ainsi devenue un lieu patrimonial plus que mémoriel ». Concernant la période contemporaine, outre l'étude déjà cité de la bataille de Dixmude, deux contributions de Christian Bougeard sur la Seconde Guerre mondiale, l'une centrée sur l'intérêt stratégique de la Bretagne et l'autre sur le maquis et la bataille de Saint-Marcel, concluent l'ouvrage. Le schéma centré sur la " bataille " dans son unité de temps et d'action est forcément abandonné, mais les études de la Bretagne en guerre restent particulièrement intéressantes, avec une attention particulière au volet mémoriel et muséographique.

En conclusion, même si on peut regretter que certains choix méthodologiques ne soient pas plus clairement assumés, la mise en avant de la critique des sources, l'intérêt et la rigueur historique de l'ensemble des contributions font de cet ouvrage utile et lucide une référence nécessaire pour l'étude de la Bretagne en guerre et des récits qui s'y sont construits. 